Cinémas

Revue d'études cinématographiques

Journal of Film Studies

\title{
Le film et le temps braudélien
}

\section{Michèle Lagny}

Volume 5, numéro 1-2, automne 1994

Le Temps au cinéma

URI : https://id.erudit.org/iderudit/1001002ar

DOI : https://doi.org/10.7202/1001002ar

Aller au sommaire du numéro

Éditeur(s)

Cinémas

ISSN

1181-6945 (imprimé)

1705-6500 (numérique)

Découvrir la revue

Citer cet article

Lagny, M. (1994). Le film et le temps braudélien. Cinémas, 5(1-2), 15-39.

https://doi.org/10.7202/1001002ar
Résumé de l'article

À partir de deux exemples, Les Camisards d'Allio (1972) et Stavisky de Resnais (1974), cet article analyse, à travers le fonctionnement de la temporalité filmique, la compétence particulière du cinéma à rendre l'enchevêtrement des temps de l'histoire, dont Braudel a montré le premier la complexité. Le film peut soit, comme Braudel, conserver une forme épique et globale au récit historique, soit, comme le voudrait Rancière, suggérer à l'historien comment raconter « autrement » l'histoire fragmentaire et morcelée d'un monde contemporain qui n’a plus de grands récits mythiques fondateurs. 


\title{
Le film et le temps braudélien
}

\section{Michèle Lagny}

\begin{abstract}
RÉSUMÉ
A partir de deux exemples, Les Camisards d'Allio (1972) et Stavisky de Resnais (1974), cet article analyse, à travers le fonctionnement de la temporalité filmique, la compétence particulière du cinéma à rendre l'enchevêtrement des temps de l'histoire, dont Braudel a montré le premier la complexité. Le film peut soit, comme Braudel, conserver une forme épique et globale au récit historique, soit, comme le voudrait Rancière, suggérer à l'historien comment raconter "autrement " l'histoire fragmentaire et morcelée d'un monde contemporain qui n'a plus de grands récits mythiques fondateurs.
\end{abstract}

\begin{abstract}
Using two examples, Allio's Les Camisards (1972) and Resnais' Stavisky (1974), this article analyses, through the functioning of filmic temporality, the special ability of cinema to render the intertwining of historical times whose complexity was first pointed out by Braudel. Film may either, as in Braudel, maintain the epic, global form of the historical account, or as Rancière suggests, show the historian an alternate way to recount the fragmentary, piecemeal history of a contemporary world which no longer has great myths of origin.
\end{abstract}

Mon propos ici sera de voir comment le film répond au défi lancé au récit par la démultiplication des temps de l'histoire, inventée par Braudel. Dans le vieux débat sur le rapport de l'histoire au récit, en effet, la question du temps est cruciale : c'est la seule spécificité de l'histoire, par rapport aux autres sciences sociales, que de travailler sur les changements dans le temps; et 
c'est justement cette spécificité qui lui interdit de construire des modèles théoriques fixes et qui l'oblige à inventer des procédures d'analyse et, plus encore, des ruses d'exposition qui instruisent le lecteur, non seulement sur la genèse et l'érosion des sociétés, mais surtout sur leurs mécanismes; qui contraint l'historien à expliquer en racontant, à combiner des formes "littéraires " avec des prétentions scientifiques, dit Rancière dans Les Mots de l'histoire.

Poser la question ainsi, c'est donc partir d'un triple postulat: d'abord, que "l'histoire est fille du récit", selon la vigoureuse formule de l'historien François Furet; ensuite, que le récit, à condition qu'il ne soit pas linéairement conçu, est le moyen de " refigurer le temps" dans sa paradoxale complexité, comme le suggère Ricœur dans Temps et récit; enfin, que le cinéma est, grâce aux pouvoirs de l'image-son, capable de construire le "récit étendu» que réclame l'histoire. Dans un premier mouvement au moins, puisqu'une autre question serait de se demander si, et comment, le récit filmique peut rendre compte de toutes les histoires, de tous les moments de l'histoire.

\section{Temps et récit historique}

La notion de temps historique est désormais pour tous celle que Braudel a imposée en termes de "multitemporalité": le temps de l'histoire n'est ni linéaire, ni continu, ni homogène comme le laisse supposer la chronologie qui sert de fil directeur au discours historique. Il est pluriel; plus exactement, dans l'épaisseur du temps qui se déroule, se différencient plusieurs flux, dont les rythmes ne concordent pas. La préface à la première édition de la thèse sur La Méditerranée, le texte fondateur, opposait la longue durée de l'éco-histoire ou des mentalités et le récitatif cyclique des transformations économiques aux soubresauts du temps agité du politique. De fait, les historiens ne se contentent pas seulement de ces trois temps initialement définis, mais ils admettent "mille temps" intermédiaires: d'une part, chaque type de phénomène (le cinéma lui-même par exemple) évolue à son rythme propre, avec ses cadences spécifiques; de l'autre, chaque niveau de l'histoire (socio-économique, politique ou culturelle, pour citer des secteurs qui concernent le cinéma) 
fonctionne sur des rythmes différenciés: il y a une longue durée du politique comme il y a des événements dans l'éco-histoire (celle du climat par exemple) ou dans les mentalités.

L'analyse du temps historique qu'a proposée Pomian, dans L'Ordre du temps, insiste sur le fait que cette pluralité, "réelle et irréductible, et qu'il faut accepter telle quelle", fait jouer l'un par rapport à l'autre un temps quantitatif, la chronométrie (temps des horloges et des calendriers, qui permet la mesure) et un temps qualitatif, qui ne se définit qu'en termes de rapports (de contemporanéité, d'antériorité ou de postérité, de parallélisme, de divergence ou de convergence). Le temps n'est plus alors qu'une "classe de relations", celles, quantitatives et qualitatives, qui s'instaurent entre les événements spectaculaires et brusques relevés par la "chronographie", qui dessine la sphère visible de l'histoire, et les "dérives imperceptibles du fait de leur lenteur ". Ces relations se jouent à la fois en fonction de la direction de l'axe du temps (c'est un vecteur réversible, qu'on peut descendre ou remonter), de sa topologie (il peut être linéaire, cyclique ou stationnaire) et de ses rythmes (court ou long, rapide ou lent, disait Braudel avec une certaine imprécision). Le temps ne s'exprime donc pas dans le changement, il est coordination de changements d'ordres divers. La question est de savoir quelle est l'instance coordinatrice qui permet au temps historique d' "émerger à la représentation ".

La chronologie, conçue comme échelle conventionnelle autorisant l'organisation de l'ordre du temps et la mesure des durées, se voit le plus souvent attribuer ce rôle. Certes, grâce à elle, on peut mettre en relation, en évaluant les synchronismes et les écarts, les différents tempos des phénomènes historiques, et donc les réarticuler, réalisant ainsi une sorte de réunification temporelle. Mais en fait, la chronologie n'est qu'un indicateur et ne peut avoir aucune fonction active, puisque, comme le souligne Pomian, elle n'a pas de "programme ", elle ne prévoit rien; la véritable instance coordinatrice, c'est le mode d'exposition choisi par l'historien, en d'autres termes la forme du discours historique, qui reste tributaire du récit puisqu'il décrit toujours quelque chose qui change entre un début et une fin. 
Or, le récit historique a été vigoureusement contesté, précisément pour une large part à cause de la reformulation du temps historique : si celui-ci n'est pas linéaire et continu, s'il fonctionne à différentes vitesses en différentes strates, il ne peut plus être l'axe unique sur lequel se disposent et s'articulent les uns par rapport aux autres les événements qui font l'histoire. La question de l'événement a été au centre du débat sur l'histoire-récit, accusée de n'être qu'histoire événementielle. Le qualificatif était une insulte sous la plume des historiens des "Annales ", grâce à qui l'histoire est devenue topographie de phénomènes massifs; l'évolution lente des structures socio-économiques, les stagnations et mutations des mentalités relèguent à l'arrière-plan ce qui occupait naguère le devant de la scène: les grands hommes, les conflits politiques. De l'histoire-problème définie par Furet comme "intellectualiste, construisant explicitement ses données à partir de questions conceptuellement posées ", à l'histoire de longue durée, structurale, donc plus descriptive que narrative proposée par Braudel, le récit fondé sur une succession d'événements était disqualifié.

Le refus de l'événement et de l'histoire comme récit repose en fait sur un double malentendu. D'une part, la nouvelle histoire a davantage critiqué le primat du politique dans le récit historique qu'elle ne s'est interrogée sur les possibilités expressives de la forme narrative: il y a confusion entre histoire politique et une histoire-récit assimilée à l'histoire-bataille. D'autre part, les critiques acharnés de l'histoire-récit ont une vision très simplifiée de la forme narrative: celle qui considère l'événement comme un "point nodal ", comme le lieu propre de l'historicité, et le développement historique comme l'articulation de ces événements dans l'avant, l'après ou le simultané (qui doivent donc être très précisément évalués sur le mode chronologique), avec une tendance irrépressible à la logique de la consécution-conséquence, c'est-à-dire au postulat affirmant que la cause d'un fait donné se trouve dans les faits antérieurs. Cette attitude polémique, qui a d'ailleurs été dénoncée par Paul Veyne il y a vingt ans ${ }^{1}$, engendre une conception réductrice du récit historique et le condamne à la linéarité, bien que peu d'historiens sérieux s'en soient tenus là : ils distinguaient souvent plusieurs faisceaux de 
causes, renvoyant, d'une manière implicite, à différents niveaux de temporalité.

La vraie question qui se pose est de savoir quelle place et quel statut donner à l'événement, et comment trouver le mode narratif qui fasse la part des lenteurs de la longue durée, des rythmes cycliques, des crises, comme le réclamait Braudel lorsqu'il insistait sur le caractère polyphonique de l'histoire et sur la nécessité de l'exposer sur un mode quasi musical. Par une étude très serrée, à la fois admirative et un peu méchante, des procédés d'exposition dans La Méditerranée, Ricœur montre que la cohérence de l'histoire y repose sur un récit construisant une "intrigue virtuelle", avec la chronologie comme épine dorsale des temps multiples ${ }^{2}$. De fait, derrière Braudel, dont le philosophe admire la capacité à réenraciner l'événement politique dans les structures et les conjonctures, beaucoup d'historiens ont su retrouver celui-ci, en le traitant comme "partie émergée de l'iceberg", comme symptôme permettant de voir affleurer des transformations en profondeur qui apparaissent mal dans les textes-traces dont nous disposons. À cette analyse symptomale correspond une nouvelle forme de récit: dans la mesure où elle met en évidence, sous formes de séquences narratives, des réseaux de relations entre des "événements" qui, pris isolément, restent incompréhensibles, elle conserve la mise en scène de moments articulés entre eux par des "intrigues"; mais elle assume, selon l'expression de Michel de Certeau, une "écriture feuilletée " qui, grâce à différents artifices de dispositio et de figures rhétoriques, lui permettent de rendre compte, à la fois, de sa démarche propre (attester et expliquer, en même temps que raconter) et de la pluritemporalité.

Précisément dans Les Mots de l'histoire, Rancière observe que l'utilisation des "temps de l'histoire et du discours" par Braudel n'est pas conforme à la règle ordinaire, telle que la définissait Benveniste: de fait, l'historien emploie souvent, dans son récit, le présent ou même le futur, qui, pour le linguiste, appartiennent à l'ordre du discours et non à celui de l'histoire. D'une part, cela permet, en déréglant l'opposition traditionnelle, de " construire un récit dans le système du discours", donnant ainsi au récit "des pouvoirs d'assertion analogues à ceux du discours"; 
parce qu'il n'enchâsse plus le récit dans ses explications, Braudel rend les deux plans indiscernables: ainsi l'histoire peut-elle et garder sa forme narrative et justifier de sa scientificité. D'autre part, du point de vue qui nous intéresse ici, l'utilisation du présent permet d'associer le temps de la longue durée et celui de l'événement qui coexistent dans le même moment. Par exemple, dans le fragment de texte choisi par Rancière, "le temps de la règle" ("les épidémies sautent à pieds joints d'une masse d'hommes à une autre masse d'hommes") entre en coalescence avec le temps de l'événement, indiqué par la phrase suivante: " [L'ambassadeur] que le grand-duc de Toscane envoie en Angleterre passera par Boulogne [...], non par Calais où la peste vient de s'infiltrer" (Rancière, p. 33-34).

De fait, cette "coalescence des temps" reste tributaire de ce que Rancière appelle des "tours littéraires", qui vont au-delà des "tours stylistiques", comme le suggère l'aspect fictionnel des pages finales de La Méditerranée, où l'historien pénètre au présent dans le bureau du roi mort. Cette observation impose de confronter, à la suite de Ricœur, temps historique et temps de la fiction; c'est un point que je n'aborderai pas dans le présent article, mais qui est fondamental pour le cinéma, puisque beaucoup de films historiques combinent, en proportions variables, des faits, sinon vrais du moins attestés, et des éléments fictionnels ${ }^{3}$.

Ce n'est pas le lieu ici d'analyser les formes écrites de l'exposition historique, mais c'est le moment de voir ce que le cinéma apporte dans ce domaine. Même si par différents moyens, celuici est capable de suggérer l'avant et l'après, il se fonde sur la succession d'images photographiques qui ont pour trait fondamental de toujours "montrer" au présent ${ }^{4}$ des instants du passé et donc de favoriser ces effets de coalescence du temps long et du temps court, qui sont chez Braudel liés à une ruse d'écriture, grâce à l'emploi non réglementaire des temps verbaux. Surtout, la caractéristique du film est d'associer deux modes de construction de la temporalité: celle du récit, au sens commun du terme, où la narration agence les événements diégétiques dans un ordre et avec une durée spécifique, largement explorée par la sémiologie d'inspiration genettienne et metzienne; celle de l'image-son qui est elle-même temporalisée, puisque le mouvement engen- 
dre la durée, et que le montage produit le rythme. L'image-son n'est pas homogène, mais elle joue sur des intervalles qui se manifestent à différents niveaux: celui de l'image visuelle et celui de l'image sonore, qui peuvent miser sur la discontinuité autant que sur la continuité (jump cut à l'image, interruption du son); celui de leur association, qui peut produire des dissonances autant que des assonances (faux raccords de montage, décalages entre l'image et le son, comme entre plusieurs espaces dans l'image, entre différents plans sonores). Les jeux plastiques et sonores peuvent donc au cinéma susciter des effets de temps pluriels. C'est bien entendu de l'image-temps deleuzienne que je veux parler ici, et dont il faudrait étudier le fonctionnement dans la perspective de la nouvelle histoire et de sa pluritemporalité; en particulier à travers les films qui, rompant avec la succession empirique des événements, celle de la chronologie, seraient capables de proposer directement l'épaisseur du temps dans toute sa paradoxale complexité et dans toute son ambiguïté. Trop vaste programme. Je me contenterai d'un exemple pour examiner la manière dont le cinéma peut produire ce récit polyphonique que réclamait Braudel.

\section{Pluritemporalité dans Les Camisards}

À vrai dire, un premier problème surgit : à examiner des listes de titres de films historiques, on s'aperçoit que quand le film se mêle de devenir historien, il se nourrit surtout d'événements politiques (guerres, révolutions ou autres "affaires" d'escroquerie). Même s'il ne se contente pas de ressusciter les "grands hommes" (héros ou antihéros) rejetés par l'histoire braudélienne, il aime tout ce que la nouvelle histoire déteste: raconter des événements du temps court. Essentiellement parce qu'il est avant tout spectacle: il ne peut pas se contenter de "planter le décor", ce qui demeure pour une large part l'activité des historiens qui doivent expliquer les processus complexes à l'œuvre derrière les événements; s'il peut donner à l'événement un arrière-plan qui suggère l'épaisseur du temps en histoire, il ne peut pas laisser vide la scène de l'image".

Les Camisards de René Allio (1972), que j'ai choisi parce qu'il évite le héros individuel, raconte la réaction des protestants des 


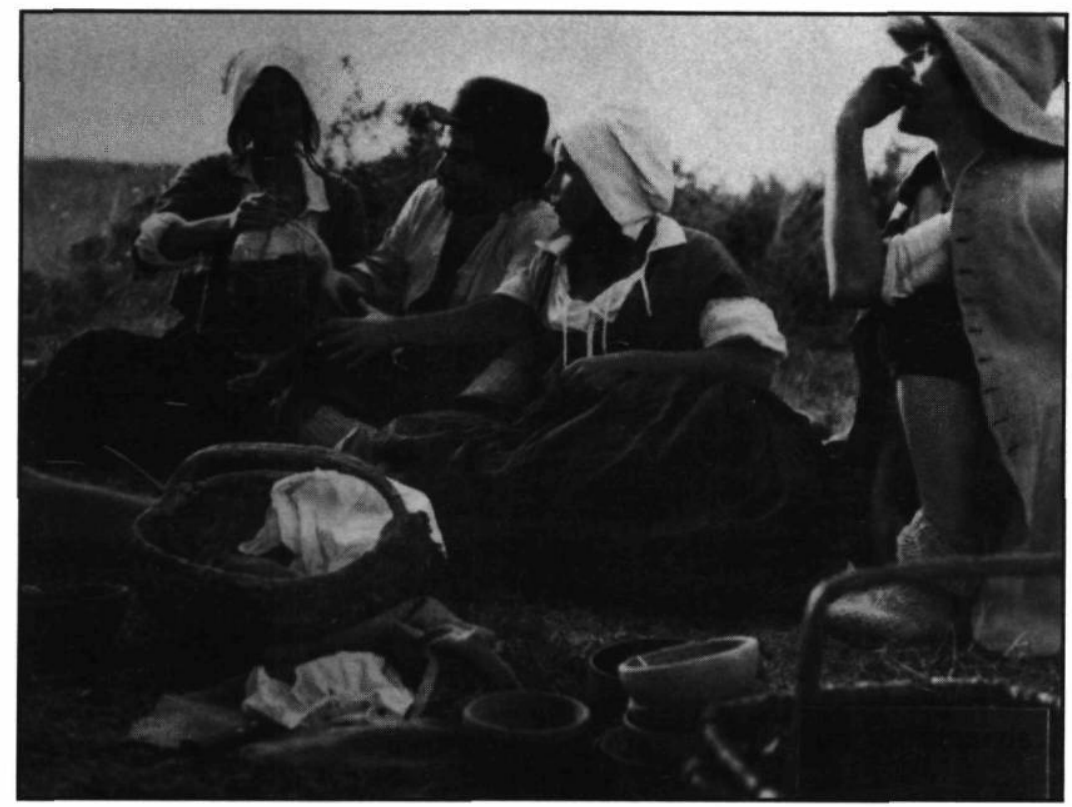

\section{Les Camisards de René Allio (1972)}

Collection Cinémathèque québécoise

Cévennes contre la répression consécutive à la révocation de l'édit de Nantes. Le temps dominant est ici le temps "court ", celui d'un conflit qui, même si ses motifs sont religieux, oppose deux groupes sociaux; mais, derrière l'épopée des camisards, Allio cherche à retrouver, d'une part, la signification de leur révolte dans les cycles des "émotions" paysannes, et d'autre part, l'enracinement de la vie paysanne dans le temps long de la nature. La coalescence des temps est produite de plusieurs manières; je n'en retiendrai ici que quelques-unes, en dissociant, par commodité, les niveaux de temporalité étroitement imbriqués dans le texte filmique.

Le temps court de l'événement se marque dans la présentation des épisodes du conflit ${ }^{6}$. Après l'exposition de la situation, les points forts sont la répression qui suit le meurtre du subdélégué de Languedoc (1), puis l'attaque du village de Vergnas qui répond à cette répression (2), enfin l'attaque du Collet de Dèze, suivie par la bataille de Champdomergue (3), où les troupes royales affrontent sans succès les insurgés qui se sont regroupés avec d'autres révoltés. L'histoire se termine sur l'écrasement 
(temporaire) des premiers camisards à Thémelac près du pont d'Anduze, où sont exposées leurs têtes coupées. On ne peut pas faire développement diégétique plus simple, avec ses trois épreuves et son échec final, ni histoire plus événementielle.

Mais cette histoire n'est pas linéaire, et son temps n'est pas homogène. D'abord, l'utilisation d'un procédé très courant mais très efficace, celui de la voix off d'un narrateur qui est aussi un personnage diégétique représenté dans le film, produit un premier effet de différence temporelle. Il s'agit du personnagenarrateur Jacques Combassous, qui commence à raconter son histoire, en relation avec la révolte, après environ un tiers du film. En voix intérieure, tout en suivant un maigre cortège d'enterrement, il se reproche d'avoir, durant son mariage avec la jeune femme qu'il vient de perdre, cédé à la tentation d'obéir au roi, et d'avoir fréquenté l'église, malgré ses convictions protestantes. On ne sait pas quand se passe cet enterrement, qui pourrait être en flash-back sans que le spectateur puisse en être certain. Le récit off continue un peu plus tard dans le film lorsque Combassous indique qu'il est parti de chez son frère à la miaoût 1702, pour entrer au "désert ". À partir de là, sa voix commente les différents épisodes événementiels représentés, en indiquant leurs dates et leurs durées approximatives, jusqu'à "quelques jours après» le 23 octobre, où les révoltés sont écrasés. Lui-même survit, et le film se termine sur l'affirmation de sa résolution de rejoindre d'autres groupes de camisards pour continuer la lutte. Cet emploi de la voix off a plusieurs effets : d'abord, Combassous figure l'historien qui date et explique un certain nombre de motivations de la révolte de l'été 1702; il localise l'événement, tout en lui donnant son sens. Mais il est lui-même présent-absent dans une double temporalité: son récit off renvoie à un "avant " et à un "après " de l'événement, et met au passé ce qui paraissait actuel dans le film, l'insérant ainsi dans une durée plus longue. Dans la durée de la résistance protestante, qui persiste au-delà d'un été, au-delà de la "guerre des camisards " (1702-1704), puisqu'elle s'étend sur un siècle, entre 1685 , où les premières "assemblées du désert " répondent à la révocation de l'édit de Nantes, et 1787 où l'édit de Tolérance rend leur statut civique aux réformés. 
Par ailleurs, la structure narrative d'ensemble fait se succéder de courtes séquences mal reliées chronologiquement, qui, à la fois, produisent des effets de simultanéité et soulignent le conflit. L'"intrigue " est dialectiquement construite: chacun des événements qui se succèdent, et dans une large mesure s'enchaînent, sont systématiquement présentés comme provoqués par l'antagonisme de classe et montés en alternance, ce qui atténue considérablement la linéarité du récit, sans l'effacer tout à fait. L'ouverture du film met en place les principes de ce discours-récit en s'organisant de manière circulaire: à une réunion obligatoire à l'église (où le curé menaçant fait l'appel) répond l'assemblée secrète des protestants qui écoutent un jeune prêcheur inspiré. La mise en scène spatiale des deux assemblées est identique (à cette différence près cependant que les places sont inversées: par rapport aux fidèles, qui sont les mêmes, le curé, qui représente le pouvoir temporel, est à gauche de l'écran; le prophète, qui symbolise le pouvoir spirituel, à droite). Entre ces deux moments, qui pointent les enjeux du conflit, une séquence, elle-même trouée en son milieu par une ébauche d'alternance, dépeint la table du déjeuner, "lieu d'assemblée" de la classe dominante (prêtres, militaires et nobles, réunis sous la protection du pouvoir royal, chez le subdélégué). Discours répressifs et bavardages futiles s'y entremêlent, qui sont interrompus par une excursion filmique dans la campagne, où des déserteurs affamés tuent le chien de pauvres braconniers. Les Camisards se développe ensuite sur une représentation alternée de la vie des protestants, paysans sous surveillance ou révoltés assemblés au "désert", et de celle des nobles protégés en leurs châteaux d'une part; entre cette vie au quotidien et les moments clés de la révolte ou de la répression de l'autre ${ }^{7}$. En affichant le conflit de classes par la structure en alternance, le film rattache la résistance rurale du "désert " à ce mouvement social d'ensemble qu'analyse Le Roy Ladurie dans Les Paysans de Languedoc, et qui montre la révolte camisarde comme maillon dans une chaîne de révoltes et de rébellions qui, du XVI ${ }^{\mathrm{c}}$ au XVIII ${ }^{\mathrm{e}}$ siècle, "disent les revendications des paysans pauvres, des journaliers, des artisans, des ouvriers, pour une vie meilleure" alors que, en un cycle de quelques siècles, le capitalisme commercial remplace le mode de production féodal. 
Ainsi, derrière l'événement raconté par la diégèse s'insère ici, par la combinaison du récit off et de l'alternance narrative, le rythme plus lent du temps socio-économique, tandis que le temps de la "longue durée" se trahit à l'image. Dans la structure du film, d'assez longues pauses descriptives de la vie des champs, d'abord, de celle des insurgés dans la nature, ensuite, viennent suspendre le récit du conflit, sans que des indications précises de dates soient données; c'est seulement leur place dans le défilement filmique qui nous permet de les situer avant ou après tel événement, mais on sent bien l'effet de "contemporanéité", plus que de simultanéité: bien que se produise un événement exceptionnel, les paysans vivent comme ils l'ont toujours fait. Ainsi de la scène des moissons entre les épisodes 1 (assassinat du subdélégué) et 2 (attaque de Vergnas), ou de la préparation du repas des révoltés dans une bergerie où ils se sont réfugiés, suivie de la lessive et du bain dans le torrent un peu plus tard. Ces éléments de la vie de tous les jours (travail et repos, cuisine et repas, lessive et bain, à la fois hygiénique et ludique) marquent le rapport de la vie paysanne à la longue durée, au temps de la nature, rythmée très simplement par l'alternance des jours et des nuits.

Certes, le film maintient la trace de l'événement (surveillance de moissons par les soldats, irruption de quelques militaires brutaux pendant le bain). Mais, alors que les paysans protestants sont décrits longuement, dans des cadres larges qui associent étroitement les hommes (et les femmes) au paysage, les militaires n'apparaissent qu'épisodiquement (dans les images, sinon dans le récit) et sont cadrés soit de près, soit comme perdus dans une nature dans laquelle ils "font tache", notamment à cause des couleurs brutales de leurs uniformes. Ensuite, justement, parce que les costumes du peuple, aux teintes en camaïeu de brun, sont non seulement parfaitement (trop parfaitement) en harmonie avec le milieu sec et austère des Cévennes, mais encore perçus comme de "longue durée". Je m'explique: alors que les vêtements des nobles sont presque directement référés à des représentations d'époque (les gravures d'Abraham Bosse sont aisément repérables derrière $\mathrm{M}^{\mathrm{me}}$ de Villeneuve, $\mathrm{M}^{\text {lle }}$ de Vergnas et leurs admirateurs), ceux des paysans sont sans âge. On y 
reconnaît certes des réminiscences de Le Nain ou de Chardin (donc approximativement d'époque), mais il pourrait aussi bien s'agir de traces de Millet ou de Van Gogh ${ }^{8}$; ce sont en fait des superpositions de représentations assez proches les unes des autres, empêchant toute "datation" précise des paysans, qui appartiennent à ce "long moyen-âge " qu'un historien comme Le Goff prolonge jusqu'au milieu du XIX $x^{e}$ siècle, jusqu'à la révolution industrielle. On pourrait aussi penser à la longue durée à propos des décors, en sachant que les plans ont été tournés dans la montagne cévenole de la fin des années soixante, certes transformée depuis le début du XVIII ${ }^{e}$ siècle, mais assez "immuable" pour être reconnaissable comme "toujours la même malgré les changements", alors que les espaces (jardins et salons) des nobles sont irrémédiablement datés. Ici, par le traitement du profilmique, mais aussi par ses cadrages et les relations entre les premiers et les arrière-plans, c'est l'image elle-même qui, grâce au jeu sur l'espace, suggère les permanences de longue durée.

Tous ces "artifices d'exposition" font entrer en coalescence trois moments au moins: celui du passé raconté, celui du récit produit par le mémorialiste qui a servi de "source" à l'historien (le camisard Bonbonnoux, modèle de Combassous, qui commence à écrire son témoignage à partir de 1730) et le présent du moment où est fait le film vers 1970 (décor et costumes). Ils entrelacent, tout en maintenant leurs écarts, la longue durée de la vie paysanne (ou plus largement de la vie du petit peuple) et l'événement de la révolte camisarde, inclus dans la lignée des "jacqueries ", des protestations paysannes (ou plus largement populaires) contre l'oppression, qui participent du temps "des destins collectifs et des mouvements d'ensemble" braudéliens et auxquelles répondent en écho lointain les utopies post-soixante-huitardes. En jouant sur les alternances, les ruptures de montage et les écarts entre bande-son et bande-image, qui viennent délinéariser le récit, mais aussi sur le profilmique, la construction de l'espace et le statut présent-passé de l'image-son, Les Camisards, comme la thèse de Braudel, à la fois préserve la multitemporalité et réussit une intégration forte, une articulation en profondeur.

C'est justement à propos de cette cohérence braudélienne qu'il faut s'interroger. Le film d'Allio pourrait se laisser lire de la 
même manière que la thèse de Braudel, que Rancière analyse comme la dernière forme de discours-récit historique capable de synthétiser les temps de l'histoire. Bien qu'écartelée entre différentes temporalités, l'histoire y est encore scénarisable et elle fait sens. L'événement n'est qu'une manifestation symbolique, qu'on peut prendre comme "modèle" dans le contexte du contrat politique rempli par le film, comme par tout récit historique: dans notre cas, expliquer la valeur de la révolte. La nouvelle histoire a inventé la notion de "mentalités", qui cimente la cohérence de l'histoire grâce aux structures symboliques des agents qui la vivent et la font. Ainsi dans notre film, les groupes en conflit disposent des structures symboliques de l'Ancien Régime, avec ses modèles politiques (la monarchie) et ses enjeux métaphysiques (la religion). Par ailleurs, Rancière insiste sur le fait que l'écriture du "discours-récit " braudélien tient sa cohésion de sa forme épique inspirée de récits fondateurs, qui rendent vrai le récit historique en le fondant sur le mythe. Ainsi, Braudel donne à La Méditerranée, qui remplace comme sujet d'histoire le roi Philippe II, son unité et son caractère, comme à un "quasi-personnage", en faisant se recouvrir un espace matériel (la mer) et un espace de discours: le «lieu originaire de tout récit d'espace: l'Odyssée" (Rancière, p. 173). De même, la valeur "mythique" du geste des camisards tient à ce qu'il fonde le logos, le discours de sa révolte, dans un muthos, Dieu qui parle par la bouche des prophètes. Elle tient aussi à ce que le film donne au récit sa vérité et à l'histoire son sens, par le même procédé que Braudel : l'histoire des protestants des Cévennes s'enracine dans un lieu (le "désert" rural) et s'appuie sur le Livre, la Bible, expression de la parole de Dieu, à laquelle se réferent directement les réformés en général et les prophètes dans le film en particulier (Mazel par exemple récite un verset du psaume 119: "Comme j'aime ta loi, Seigneur, / Tout le jour je la médite $[\ldots] »)$.

\section{L'histoire postbraudélienne: Stavisky}

Cette analyse vaut surtout pour les périodes "médiévale» et "moderne", au sens chronologique des historiens, qui ont surtout sollicité l'attention de la nouvelle histoire. En revanche, 


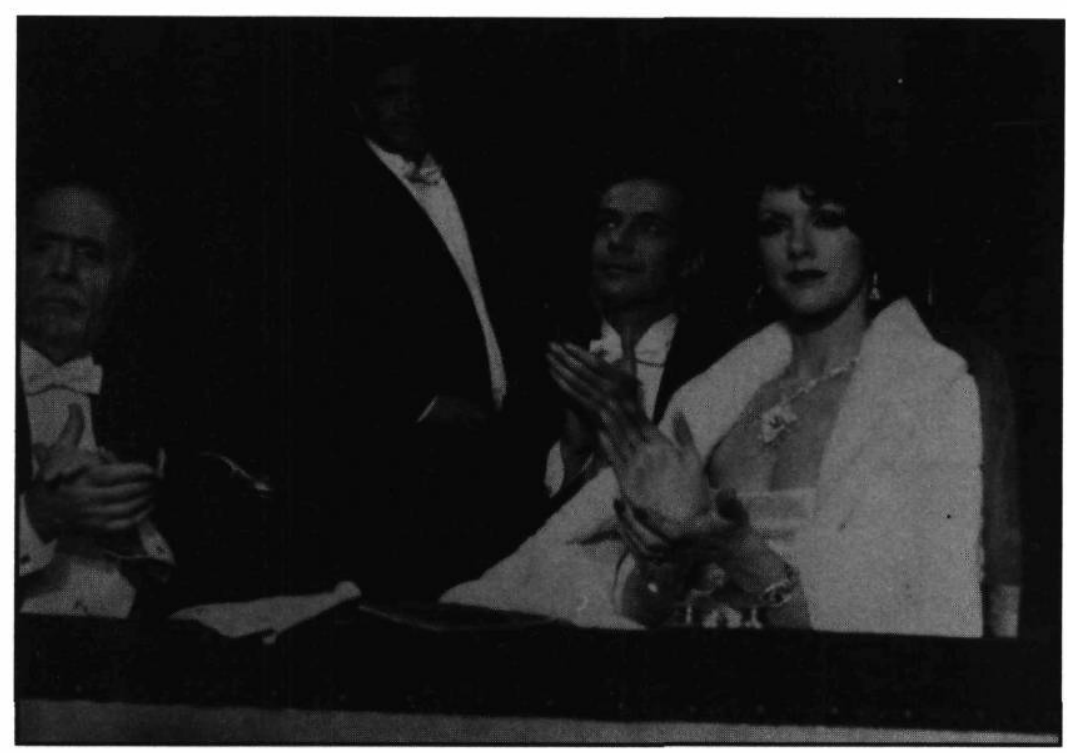

\section{Stavisky d'Alain Resnais (1974)}

Collection Cinémathèque québécoise

pour la période contemporaine, l'histoire est devenue une histoire éclatée, où l'articulation entre les contraintes de la longue durée et les effervescences de l'événement ne se fait plus. Pour Rancière, c'est justement parce que, depuis l'âge à la fois de la grande industrialisation et de la démocratie, depuis l'âge des "masses", les structures symboliques anciennes et devenues inadaptées ont été détruites, mais pas remplacées. C'est aussi parce qu'il n'y a plus de modèle pour régler l'organisation du discoursrécit: il n'y a plus de Livre, ni de Loi, qui puisse servir de fondement (d'origine) au récit, en même temps que de centre (de lieu d'articulation) à la diversité des rythmes temporels. Prenant en compte cette rupture profonde, l'histoire braudélienne a cédé la place à l'histoire en miettes, qui juxtapose, au risque de se perdre dans une pseudo-scientificité descriptive et illisible, les descriptions souvent chiffrées d'une socio-histoire, voire d'une ethnohistoire, des analyses sur les déterminations économiques ou culturelles et le récit heurté d'événements bavards et répétitifs, dont la "nécessité» est souvent absurde, superficielle, contradictoire. Pour éviter cet écueil, il lui faut construire une nouvelle 
poétique, une nouvelle forme de récit non plus "étendu" mais au contraire capable de rendre intelligibles les écarts qui la constituent en marquant l'entrelacement des temps sans vouloir faire la synthèse de l'hétérogène. Le nouveau récit historique devrait jouer de la déstructuration du temps linéaire en suggérant que l'intelligibilité de l'histoire est dans les mécanismes cachés, dans ce qui ne se dit pas, ne se représente pas, alors que le bavardage des événements a pour fonction de nous brouiller la vue.

Je n'analyserai pas en détail ce type de récit au cinéma, puisque ce serait entrer dans un tout autre sujet que Deleuze a balisé dans son étude sur la "narration cristalline" et les "puissances du faux ${ }^{9}$ ". Prenons tout de même un exemple rapide, avec le Stavisky de Resnais, sorti en 1974. Ce qui frappe dans ce film, c'est l'allure chaotique du temps chronologique dont la linéarité est détruite par les alternances: l'une entre la vie de Trotsky, en résidence surveillée en France au moment du scandale financier qui ébranle le monde politique entre 1933 et 1934, et celle de Stavisky (effet de simultanéité); l'autre produite par des flash-backs et des flash-forwards qui viennent constamment interrompre les activités de ce dernier (parcours du vecteur temporel dans un sens et dans l'autre). L'organisation du début du film est à cet égard exemplaire: il commence par l'arrivée de Trotsky à Cassis, puis fait alterner des flashes de la voiture de l'exilé sur la route qui le mène vers la région parisienne avec des images du Claridge à Paris, où Stavisky est en conversation avec un comparse, le baron Raoul, avec des retours en arrière sur le concours d'élégance de Biarritz, où sa compagne a triomphé la veille, et avec des plans sur deux inspecteurs de la Sûreté générale, qui font allusion aux recherches policières sur le passé douteux du héros. Cette structure se complique encore après quelques minutes de film, puisque, pendant que Stavisky règle le prochain spectacle de son théâtre, l'Empire, son médecin et son avocat rappellent son arrestation à Marly sept ans plus tôt, filmée en flash-back, tandis qu'immédiatement après apparaît un plan au statut temporel incertain, où un des inspecteurs témoigne, en plan rapproché et regard-caméra, sur cette arrestation. On ne comprendra que plus tard qu'il témoigne, en flash-forward, pour la commission d'enquête qui se déroulera au Palais-Bourbon en avril 1934. 
La description des événements elle-même est obscure: les fréquentes insertions de "journaux de l'époque" (aux photos truquées pour les besoins du film, puisque les visages de Belmondo et d'Annie Duperey viennent se substituer à ceux de Stavisky et de sa compagne) donnent des indications contradictoires, et le discours du principal acteur est présenté comme celui d'un menteur, d'un joueur, d'un "spectre" de théâtre. Les explications proposées restent au niveau du politique, et revêtent un aspect dont le caractère idéologique est commenté dans le film par les propos de comparses. En tirant des conclusions liées à ses propres préoccupations révolutionnaires, le jeune admirateur de Trotsky souligne l'absurdité du "récit événementiel " traditionnel que la commission d'enquête d'avril 1934 prend à son compte : "Voilà, ils vont expulser Trotsky. [...] C'est fou quand même que Stavisky ait provoqué ça. [...] Sans Stavisky, pas de 6 février. Sans l'émeute fasciste du 6 février devant laquelle Daladier capitule, pas de gouvernement d'Union nationale. Sans gouvernement d'Union nationale pas d'expulsion de Trotsky. Donc, sans Stavisky..." Tandis que le baron Raoul rappelle le point de vue du public (et des électeurs) de l'époque, comme du spectateur du film: "Je suis comme Fabrice à Waterloo, messieurs: je ne connais qu'un petit morceau de ce casse-tête chinois."

Cela ne veut pas dire que le film de Resnais en revient à une histoire-récit politique et linéaire, et de surcroît mal faite. Disons, pour simplifier, que le temps prend l'allure d'une succession d'instants discrets dont la durée est incertaine et dont les interrelations ne sont pas assurées, ce qui empêche la mise en rapport des différents niveaux de temporalité et provoque une désarticulation entre temps vécu et temps historique. Le film met ici en place, à propos d'un faussaire mondain, lui-même victime de faussaires politiques, la chaîne des faussaires deleuzienne à l'œuvre dans la description pure : montrer c'est occulter, et les mécanismes de l'histoire sont dans ce qui ne se montre pas, dans les failles et les ruptures de montage, dans le "récit éparpillé" comme derrière l'artifice de l'image photographiée "comme pour Vogue» (traits dénoncés à l'époque par certains critiques du film). Bien d'autres procédés d'exposition seraient à 
analyser : le travail du regard (regard-caméra qui interpelle directement le spectateur, ou regard médiatisé par des observateurs diégétiques armés de jumelles); le statut des différents discours des personnages secondaires sur Stavisky et sur Trotsky, toujours présentés de l'extérieur, sinon en voix off, mais avec de fréquents décalages entre la bande-son et l'image. Il faudrait aussi prendre en compte le rapport au théâtre (pour Stavisky) et à la littérature russe (pour Trotsky, qui vit "comme dans les romans de Dostoïevsky").

Ce qu'on voit ici, c'est l'expression directe du temps, dont Deleuze rappelle qu'elle met en crise la notion de vérité, puisque, toujours en devenir, le temps ôte toute stabilité au discours rationalisateur. L'histoire de Stavisky non seulement n'a aucun sens en elle-même, mais elle ne nous propose aucune "leçon". L'événement symptôme (le suicide organisé d'un escroc) n'a plus, comme dans la guerre des camisards, une valeur de symbole; il n'est plus qu'un signal. L'histoire se passe ailleurs que sur le devant de la scène, dans les coulisses de l'événement, par exemple dans l'expédition financière à Rome de l'Espagnol Montalvo qui prépare la guerre d'Espagne, et donc celle entre le fascisme et les démocraties corrompues. Elle reste en filigrane derrière le bavardage événementiel, privée de la cohérence que lui rêvait Braudel au sein des "économies-mondes" qu'il tentait de conceptualiser. Stavisky témoigne du fait que, pour "l'histoire en miettes" actuelle, fille des "écarts" que constatait Braudel, mais qu'il masquait en partie par son jeu d'écriture, les divergences l'emportent sur les convergences, et que l'histoire a perdu et son centre et son "sens", dans les différentes acceptions du terme.

Dans leur manière de marquer le trouble du temps historique, les deux films nous permettent de pointer à la fois les apports de la réflexion braudélienne sur les temps et l'écriture de l'histoire, et le rôle que peut jouer le cinéma dans la formulation du discours-récit historique. Le film est capable de montrer simultanément à l'écran, les articulant ainsi les unes par rapport aux autres, des données de longue durée et des manifestations spectaculaires, donc ponctuelles, parce qu'il peut associer le temps d'un récit linéaire, dont il faut étudier les modalités 
d'organisation "quantitative" (ordre et durée), à un temps "qualitatif", manifesté dans son épaisseur et dans la diversité de ses flux grâce à l'image-son: ainsi, on retrouve dans Les Camisards la polyphonie du récit, réclamée par Braudel pour rendre compte de la multitemporalité, et la scène centrale est occupée par une histoire dont on peut dégager la signification et la fonction. Mais le cinéma peut aussi jouer la narration falsifiante liée à l'expression directe du temps: ainsi dans Stavisky, l'allure hachurée du récit, la superficialité de l'image viennent suggérer sans les actualiser un certain nombre de relations possibles entre différents temps, sans qu'on puisse articuler les durées de l'histoire, qui reste alors virtuelle. Ce qui reste ici de Braudel, c'est la force des écarts temporels; lacunaire, fragmentaire, discontinue, cette histoire n'est ni vraie par essence (puisqu'elle ne se fonde plus dans le mythe) ni fausse, comme voudraient le faire croire les révisionnistes de tout poil (puisqu'il n'y a pas de fausseté sans vérité) : c'est dans la chaîne de questionnement qu'elle engendre qu'elle se rend lisible, compréhensible.

Je formule cette conclusion (provisoire) sur un ton d'autant plus assuré que je pressens qu'elle doit être discutée et que je vais être probablement contredite dans beaucoup de textes présentés dans ce numéro. Je compte bien sur ces contradictions pour faire avancer ma réflexion.

\section{Université de Paris III (Sorbonne Nouvelle)}

\section{NOTES}

1 Voir notamment Comment on écrit l'histoire. Essai d'épistémologie (Paris: Seuil, 1971).

2 Cette "intrigue virtuelle", ou "quasi-intrigue», est constituée par emboîtement de différentes intrigues, de différents trajets narratifs, et opère une synthèse de l'hétérogénéité du temps de l'histoire en articulant les trois niveaux temporels de l'histoire sur le temps mesurable des calendriers; du coup, la "conspiration événementielle", le retour à un récit linéaire et simplificateur, menace sans cesse la complexité des relations qu'établit l'historien entre les rythmes inégaux des phénomènes décrits (de la "part du milieu" aux "événements" et aux "hommes" en passant par les "destins collectifs et les mouvements d'ensemble»). Cette analyse n'est, à la relecture de Braudel, qu'en partie seulement pertinente: j'ai au contraire été frappée par les hiatus, les écarts qui restent parfois béants entre les niveaux temporels décrits, notamment dans les deux premiers volumes de la thèse, où la chronologie est malmenée; au moins dans La Méditerranée, la synthèse est ouverte, et la linéarité conjurée. 
3 La fonction de ces éléments fictionnels peut être double: favoriser l'identification affective du spectateur et créer des personnages métaphoriques, dont les caractères et les actions, plus ou moins symboliques, permettent de mettre en scène des idées abstraites. C'est le cas, par exemple, dans Les Damnés (1969), des membres de la famille Essenbeck que Visconti utilise pour représenter les différentes forces idéologiques en conflit dans une Allemagne qui bascule dans le nazisme. Et c'est aussi le cas, d'une manière un peu différente, dans les deux films que j'ai choisis ici et qui ne font pas exception à la règle.

4 Ou plutôt, pour suivre une remarque de François Jost, à l'imperfectif, qui caractérise le présent comme l'image, qui "passent " et "se passent ".

5 Le fait que l'événement "fasse retour ", d'ailleurs, dans l'historiographie actuelle, pourrait tenir, comme le remarquait Pierre Nora il y a déjà vingt ans dans Faire de l'histoire (Paris: Gallimard, 1974), à l'importance de l'audiovisuel dans l'information, dans l'histoire au présent.

6 Voir découpage en annexe.

7 Même les épisodes clés ne sont pas racontés linéairement: ainsi, pour l'épreuve 2 , suit-on les insurgés (attaque de la maison du curé et contre-attaque de quelques soldats, vite défaits), mais la continuité est coupée par des interruptions : les camisards chassent les gardes, volent du ravitaillement, exécutent un traître dans sa ferme, repoussent les soldats; néanmoins, entre la séquence de l'attaque et celle de l'exécution, on assiste à une scène de galanterie entre la veuve du subdélégué et le capitaine qui doit diriger la répression, mais espère capter, en même temps que ses faveurs, les biens de la dame.

8 Ce point est revendiqué par Allio lui-même, dans "Foin de bergerie" publié en introduction à "Cinémas paysans", CinémAction, n 16 (1981).

9 Voir Cinéma 2. L'Image-temps (Paris: Minuit, 1985). J'ai amorcé ailleurs, avec des études sur plusieurs œuvres de Visconti et sur La Passagère (1963), un film du Polonais Munk, une réflexion sur cette question.

\section{BIBLIOGRAPHIE}

Braudel, Fernand. La Méditerranée et le monde méditerranéen à l'époque de Philippe II. Paris: Armand Colin, 1949.

Certeau, Michel de. L'Écriture de l'histoire. Paris: Gallimard, 1975.

Deleuze, Gilles. Cinéma 2. L'Image-temps. Paris: Minuit, 1985.

Furet, François. L'Atelier de l'histoire. Paris: Flammarion, 1982.

Le Roy Ladurie, Emmanuel. Les Paysans de Languedoc. Paris: Flammarion, 1969.

Nora, Pierre (direction). Faire de l'histoire. Paris: Gallimard, 1974.

Rancière, Jacques. Les Mots de l'histoire. Essai de poétique du savoir. Paris: Seuil, 1992. Ricœur, Paul. Temps et récit. Paris: Seuil, 3 tomes, 1983-1985. 


\section{ANNEXE \\ Découpage du film Les Camisards}

\section{Exposition}

1 - Scène, intérieur jour

Petite église de village, sermon du curé aux fidèles, qui sont contraints de répondre à un appel nominal relation chrono faible

2 - Alternance intérieur jour/extérieur jour

Déjeuner dans la salle à manger du subdélégué (représentant de l'autorité royale) qui reçoit le curé, les militaires chargés de la répression des révoltes protestantes, d'un noble anciennement protestant mais rallié et sa fille

Brève interruption: scène dans la campagne où des soldats affamés tuent le chien de pauvres mendiants

Retour au déjeuner chez le subdélégué

$$
\text { suspension }
$$

3 - Continuité, extérieur soir

Les fidèles de l'église (séquence 1) écoutent le prêche contestataire d'un jeune homme

$$
\text { suspension }
$$

4 - Continuité à ellipse, extérieur nuit

Une des jeunes femmes, vue en 1 et 3 , Marie, rencontre les soldats aperçus à la séquence 2 ; ce sont des déserteurs qui rejoignent les protestants; elle les emmène dans la montagne, rencontrer une troupe de révoltés réfugiés au "désert " suspension

Première action des révoltés (1)

5 - Continuité, extérieur jour

La bande de révoltés, qui a intégré les déserteurs, attaque la voiture du subdélégué et le tue relation chronologique 
6 - Continuité, intérieur, puis extérieur jour

Retour à la séquence 1 ; le curé fait l'appel à l'église quand le lieutenant de police fait irruption, annonce le meurtre $d u$ subdélégué et fait sortir les fidèles pour interrogatoire, sous le regard du cocher qui s'est échappé

relation chronologique et faible

7 - Continuité, intérieur puis extérieur jour

Ridicules rites de condoléances auprès de $\mathrm{M}^{\mathrm{me}}$ de Villeneuve, veuve du subdélégué

suspension ou relation chronologique faible

Alternance : vie des paysans surveillés et des révoltés au désert

8 - Continuité à ellipses, extérieur jour

Scène des moissons sous surveillance des soldats; un des révoltés, le prophète Mazel, vient cependant fixer un rendez-vous pour la nuit, puis Marie qui s'est éloignée est menacée par un soldat, arrêté dans ses brutalités par le lieutenant et le curé, qui surveillent eux aussi

\section{suspension}

9 - Continuité; début récit voix intérieure off

Pendant l'enterrement de sa femme, un protestant, Jacques Combassous, explique les raisons de sa révolte

suspension

10 - Séquence en alternance

Les paysans travaillent dans la grange du curé, tandis que celuici parle avec les soldats

\section{suspension}

\section{1 - Continuité à ellipses, extérieur nuit}

Une assemblée au désert, où Abraham Mazel le prophète est inspiré de Dieu (celle qu'il a convoquée lors de la moisson, séquence 8 ?)

En revenant, l'assemblée se heurte aux soldats qui la pourchassent suspension

12 - Alternance intérieur nuit, extérieur nuit, intérieur jour

Combassous chez son frère Elie explique, en voix intérieure off, qu'il a entendu parler des révoltés 
Les révoltés blessés au désert; nouvelle inspiration de Mazel

Suite récit off, Combassous indique sa décision d'aller rejoindre les révoltés au "désert " et en donne la date, mi-août 1702

Scène de galanterie au château de Vergnas (idylle entre $M^{\text {lle }}$ de Vergnas et le lieutenant) suspension

\section{Deuxième action des révoltés (2)}

13 - Continuité, puis alternance partielle; extérieur nuit

Suite récit off, Combassous explique que les révoltés sont affamés et commente l'attaque de Vergnas

Attaque de l'église de Vergnas et des provisions entassées chez le curé

Les révoltés battent le blé; en alternance Jacques Combassous qui va frapper chez son frère

$$
\text { relation chronologique }
$$

14 - Scène, extérieur jour

Déjeuner galant entre $M^{\text {me }}$ de Villeneuve et le capitaine Poul; l'abbé les prévient de l'attaque de la nuit précédente relation chronologique

15 - Continuité

Les révoltés exécutent le paysan traître Brunet relation chronologique

16 - Continuité à ellipse

Le capitaine Poul et sa troupe à la ferme des Brunet relation chronologique

17 - Amorce alternance révoltés et soldats

Poursuite des révoltés dans la forêt, mais les soldats s'enfuient devant leur résistance (commentaire off de Combassous par deux fois, au début et à la fin : pas de date, mais permet de comprendre que de 13 à 17 se sont écoulés une nuit et un jour)

relation chronologique faible 


\section{Vie des révoltés au désert}

18 - Séquence à épisodes; intérieur nuit bergerie, extérieur nuit Vie des révoltés dans le désert (commentaire off de Combassous qui indique jonction entre troupes de Gédéon Laporte, celle que nous avons suivie jusqu'ici, et de Jean Cavalier d'Anduze, non loin de là)

$$
\text { suspension }
$$

19 - Scène, extérieur jour

Conversations entre $M^{\text {lle }}$ de Vergnas et le lieutenant suspension

20 - Continuité

Lessive et bains à la rivière: attaque brusque de quelques soldats qui menacent La Fleur, un des déserteurs qui a rejoint les révoltés, mais il réussit à s'échapper

$$
\text { relation chronologique }
$$

\section{1 - Scène}

La Fleur retrouvé par Combassous; comme il est nu, ils volent un voyageur de sa chemise

$$
\text { relation chronologique faible }
$$

\section{2 - Scène intérieur nuit bergerie}

Entrevue de Gédéon Laporte et Jean Cavalier, lisant le courrier royal qu'ils ont enlevé (commentaire off de Combassous); préparent un piège pour les troupes du roi au Collet de Dèze relation logico-diégétique

\section{Troisième action des révoltés (3)}

23 - Continuité à ellipses extérieur jour, puis intérieur soir Attaque du Collet de Dèze (commentaire off de Combassous), suivie d'un prêche de Mazel dans l'ancien temple réformé

$$
\text { relation chronologique }
$$

24 - Continuité à ellipse extérieur jour

Les révoltés dans la forêt partent, se sachant poursuivis par les troupes royales

$$
\text { relation chronologique }
$$


25 - Alternance extérieur jour

Bataille de Champdomergue, à la sortie du bois; les révoltés réussissent à se sauver, malgré la supériorité des troupes royales suspension

\section{Alternance vie des révoltés et des nobles}

26 - Scène chez $\mathrm{M}^{\mathrm{me}}$ de Villeneuve, intérieur jour

Galanterie entre le capitaine Poul et la veuve du subdélégué suspension

27 - Scène extérieur jour

séparation entre troupes de Laporte et de Cavalier (commentaire off de Combassous)

$$
\text { suspension }
$$

28 - Séquence à épisodes extérieur jour, puis intérieur nuit Marie est faite prisonnière

$$
\text { suspension }
$$

29 - Continuité extérieur jour

$\mathrm{M}^{\mathrm{me}}$ de Villeneuve attaquée par les révoltés dans la forêt suspension

30 - Scène intérieur jour

Le nouveau subdélégué reproche son inefficacité au capitaine Poul; lit une lettre de Gédéon Laporte qui donne un ultimatum de trois jours pour libérer Marie

$$
\text { suspension }
$$

31 - Continuité extérieur et intérieur jour

Retour de $\mathrm{M}^{\mathrm{me}}$ de Villeneuve après son attaque dans la forêt (suit 29, après ellipse)

$$
\text { suspension }
$$

32 -Continuité extérieur jour

Les troupes royales à l'exercice

$$
\text { suspension }
$$

33 - Séquence à épisodes extérieur jour, puis intérieur dans plusieurs lieux

Vie des révoltés, leurs différentes attaques (commentaire off de Combassous, qui donne indication de date, fin septembre à mioctobre) 
suspension

34 - Scène intérieur jour

Prise du baron de Vergnas

relation chronologique faible

35 - Scène intérieur jour

Répression à Vergnas; deux révoltés pendus après avoir dû abjurer

$$
\text { relation chronologique faible }
$$

36 - Séquence à épisodes extérieur jour dans plusieurs lieux Attaques des révoltés (commentaire off de Combassous) relation chronologique faible

\section{Épilogue}

37 - Les révoltés écrasés à Thémelac (encadrée par commentaire off de Combassous qui donne indication de date: 23 octobre) relation chronologique

38 - Séquence à épisodes en alternance; extérieur jour, plusieurs lieux

Fuite de Marie qui semble devenue folle, en alternance avec celle de Combassous qui se cache (commentaire off de Combassous à la fin qui raconte la décapitation des cadavres de Thémelac) relation chronologique

39 - Scène intérieur jour

Au château de Vergnas, le subdélégué à déjeuner se félicite de l'écrasement des révoltés

$$
\text { relation chronologique }
$$

40- Scène extérieur jour

Combassous va rejoindre Cavalier en passant par le pont d'Anduze où sont exposées les têtes coupées (commentaire off qui indique "peu de jours après" et que c'est à ce moment qu'on commença de les appeler "camisards", dans la lutte qui se poursuit) 